\title{
Mayores activos y su relación con Internet: usos y motivaciones de uso en una muestra de mayores activos portugueses
}

\author{
Roberto Martínez-Pecino \\ Departamento de Psicología Social. Universidad de Sevilla, España. \\ rmpecino@us.es \\ Alice Delerue Matos \\ Centro de Investigação em Ciências Sociais. Universidade do Minho, Portugal \\ Patrícia Silva \\ Centro de Investigação em Ciências Sociais. Universidade do Minho, Portugal
}

\begin{abstract}
The Internet is increasingly relevant in all spheres. Participating in it is a key factor for social integration. Research on information and communication technologies (ICT) has tended to focus on younger people or on inactive seniors. However, the increase in life expectancy and quality of live favours the fact that active seniors represent an increasingly significant volume of the population. This study analyses the familiarity, uses, and motivations and benefits of using the web of Portuguese university senior students with the Internet. Results derive from a questionnaire completed by 85 seniors enrolled on university courses for older people in Portugal. We present the main outcomes about their familiarity with the Internet, the uses they make of it and the main benefits of using the Web. According to the results we warn about the risk of digital divide and emphasize the potential role of university programs for the elderly to provide them with courses and training about the Internet.
\end{abstract}

Keywords: Internet, elderly people, senior university, ICT, social inclusion

\section{Resumen}

Hoy día la relevancia de Internet es cada vez mayor en todos los ámbitos. Participar en la misma es fundamental para estar integrados socialmente. La investigación sobre las tecnologías de la información y de la comunicación (TIC) ha tendido a centrarse en jóvenes o en mayores inactivos. Sin embargo, el aumento de la esperanza y la calidad de vida conllevan que los mayores activos supongan un volumen creciente y significativo de la población. En este estudio se analizan la familiaridad, usos, motivaciones y beneficios de la utilización de Internet por parte de mayores universitarios portugueses. Se administró un cuestionario a 85 personas inscritas en programas universitarios de mayores en Portugal. Presentamos los principales resultados acerca de su familiaridad con Internet, los usos que realizan de la misma y los principales beneficios de su utilización. Conforme a los resultados, se alerta del riesgo de brecha digital y se destaca la potencial utilidad de los programas universitarios de mayores para facilitar formación sobre Internet.

Palabras clave: Internet, personas mayores, universidades de mayores, TIC, inclusión social 


\section{Introducción}

La relevancia de Internet es cada vez mayor en todos los ámbitos. Participar en la misma es fundamental para estar integrados socialmente. Su importancia ha sido reconocida por organismos internacionales como el Parlamento Europeo, la Unesco, el Consejo de Europa y las Naciones Unidas (Aguaded, 2010). Los mayores representan un gran volumen de la población actual y futura. No obstante, si bien los más jóvenes han recibido gran atención por parte de la investigación científica en relación a Internet, el interés en las personas mayores ha sido más escaso (Selwyn, Gorard, Furlong y Madden, 2003) y frecuentemente ha tendido a centrarse en personas inactivas (Hernandez-Encuentra, Pousada y Gómez-Zuñiga, 2009). Sin embargo, el aumento de la esperanza y la calidad de vida favorece que los mayores activos representen un porcentaje creciente la población. La implicación de los mismos con Internet resulta fundamental para la participación e integración social.

En este estudio nos centramos en personas mayores activas inscritas en los programas universitarios de mayores en Portugal. El objetivo de este trabajo es analizar la familiaridad de los mayores activos con Internet, el uso de la misma, así como conocer los principales beneficios o motivaciones de su utilización.

\section{Metodología}

Se administró el cuestionario empleado por Martínez-Pecino, Cabecinhas y Loscertales (2011) de forma voluntaria y anónima a 85 personas pertenecientes a programas universitarios de mayores en Portugal

\section{Resultados}

\subsection{Familiaridad con Internet}

En relación a los datos referentes a la familiaridad con Internet, el $56 \%$ de los participantes afirma que utiliza Internet. Aquellos que la utilizan comenzaron a emplear en primer lugar el ordenador y, posteriormente, Internet. De este modo, llevan 10,7 años de media utilizando el ordenador y 8,03 años utilizando Internet. El 90,9\% de los que la uti-

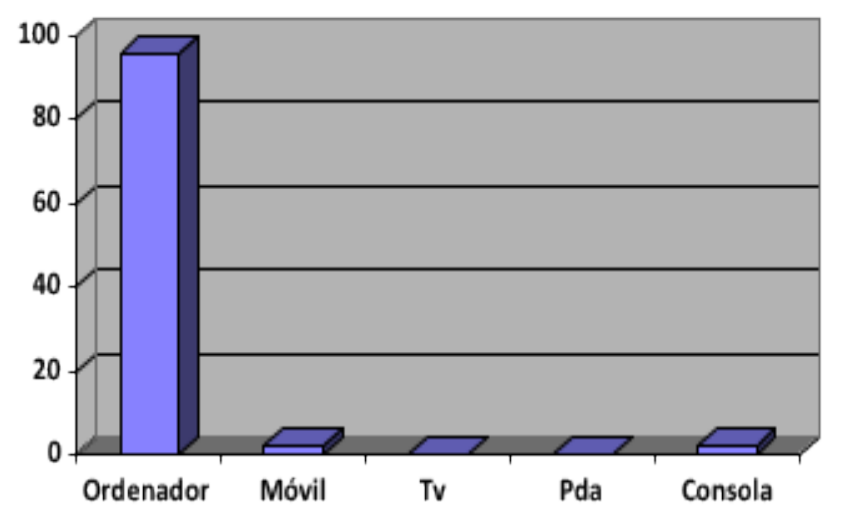

Figura 1. Forma de conexión a Internet

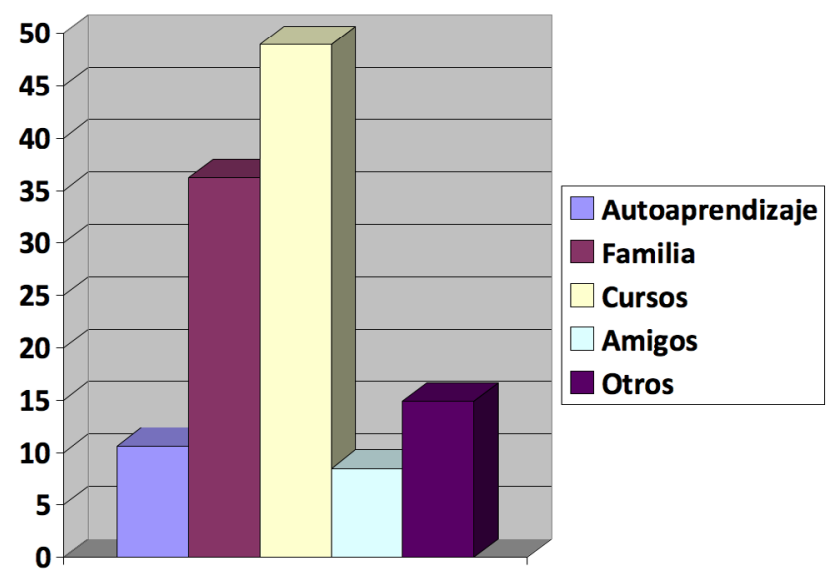

Figura 2. Forma en la que aprendieron a usar Internet expresada en porcentajes

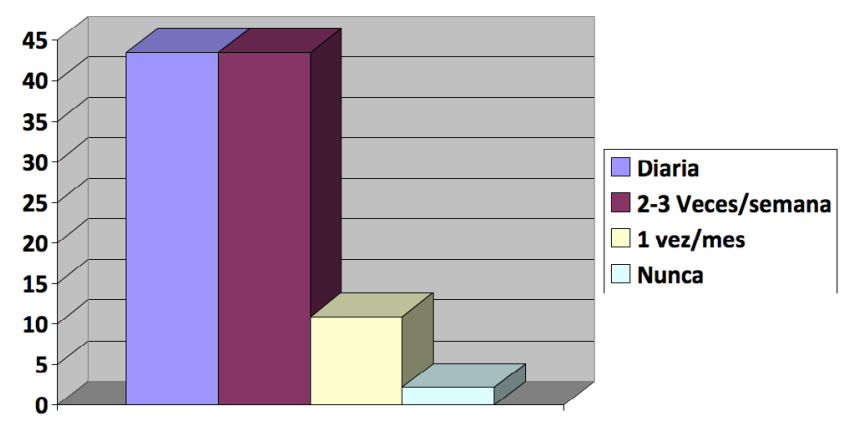

Figura 3. Frecuencia de conexión a Internet (\%)

lizan tienen conexión a Internet en casa. La forma de conexión en el 95,7\% de los casos es mediante el ordenador quedando descartados otros dispositivos como el móvil, la televisión, las agendas electrónicas (Pda) y las videoconsolas (Figura 1).

En cuanto a cómo han aprendido a utilizar Internet, los resultados indican que han aprendido a 


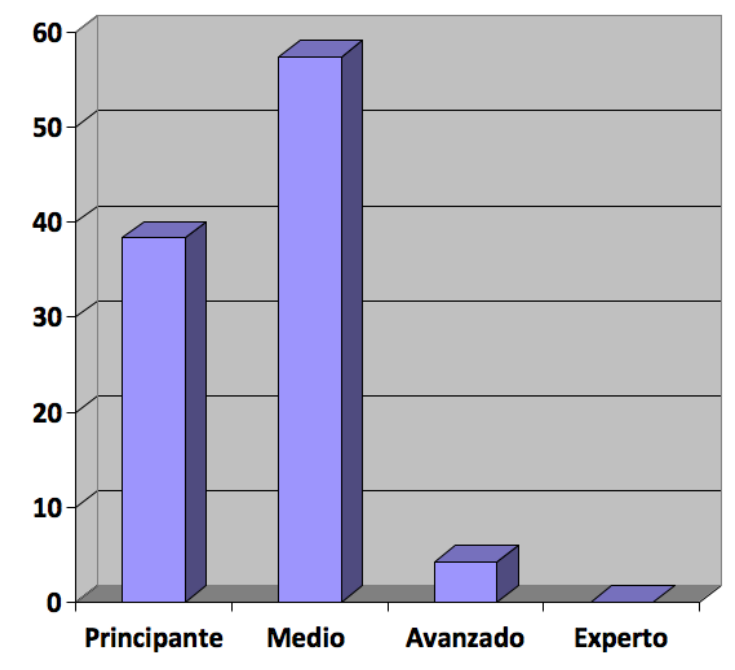

Figura 4. Conocimiento de informática (\%)

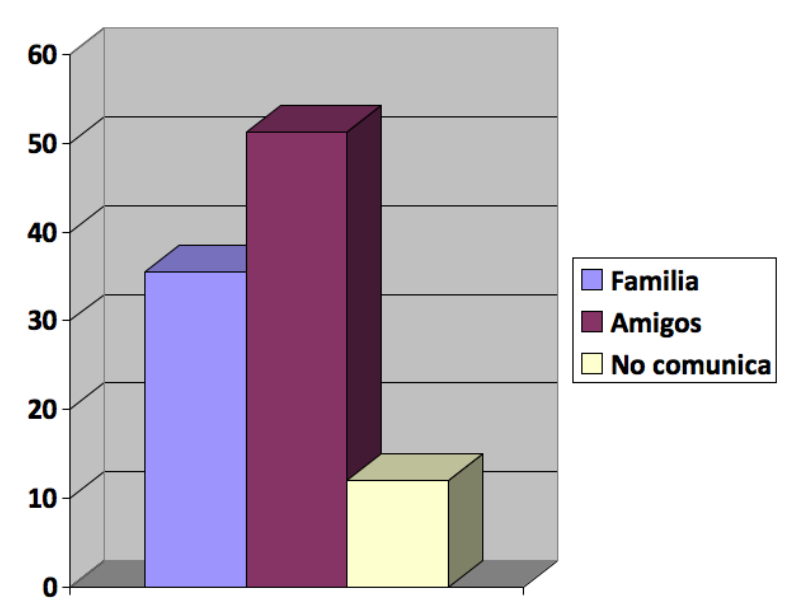

Figura 5. Personas con las que más contacta por Internet

utilizar la red principalmente mediante cursos, seguido de la familia (figura 2).

La frecuencia de conexión es diaria o de dos o tres veces por semana (figura 3 ). La valoración que realizan de su conocimiento de informática es de un nivel medio o de principiante (figura 4).

Por lo que se refiere al grado en que consideran Internet como fuente de información (principal, secundaria pero importante, secundaria pero no importante, no uso Internet como fuente de información), la valoran como una fuente secundaria pero importante de información. El $66 \%$ ha utilizado Internet para buscar información relativa a temas relacionados con la salud. Respecto a las personas con las que más contacto mantienen por Internet, la mayor parte se comunican con sus amigos, seguido de familiares (figura 5).

La red ha reducido el tiempo que dedican a ver la televisión y a dormir. Por lo que respecta a si han perdido la noción del tiempo estando en Internet, el 55,6 \% indica que perdieron la noción del tiempo algunas veces y el $28,9 \%$ indica que casi nunca.

\subsection{Beneficios y motivaciones de uso}

Respecto a los principales beneficios o motivaciones de la utilización de Internet, destacan que les resulta útil, que les ayuda a estar actualizados y que la consideran fácil de usar. No obstante, manifiestan que pueden imaginar su vida sin Internet. Por otro lado, no la destinan a conocer nuevas personas ni para encontrar gente con inquietudes similares; en la misma línea, no consideran que sea más fácil expresar algunas cosas por Internet en vez de cara a cara. Les preocupa la confidencialidad en la red (figuras 6 y 7 ).

\subsection{Usos que realizan}

Los resultados acerca de los usos que realizan de Internet muestran que la utilizan fundamentalmente para el correo electrónico, la búsqueda de información, consultar la prensa, navegar sin ningún propósito y para fines académicos y, en menor medida, para compartir fotos y gestiones con la Administración pública (figuras 8 y 9).

\section{Discusión}

Los resultados de este estudio alertan del riesgo de exclusión social que pueden sufrir los mayores activos situándose en el lado desfavorecido de la brecha digital tanto por el porcentaje de personas que la utilizan como por la intensidad y el nivel de uso, que se centra en funciones básicas. La brecha digital puede ser definida como las desigualdades existentes en relación al acceso a Internet, al grado de su utilización, al conocimiento de la misma y de sus aspectos técnicos, al apoyo social, a la habilidad para valorar la calidad de la información y a la diversidad de usos (DiMaggio y cols. 2001). Teniendo en cuenta que los participantes en el estudio señalan que su fuente principal de aprendizaje para utilizar Internet ha sido la realización de cursos, 


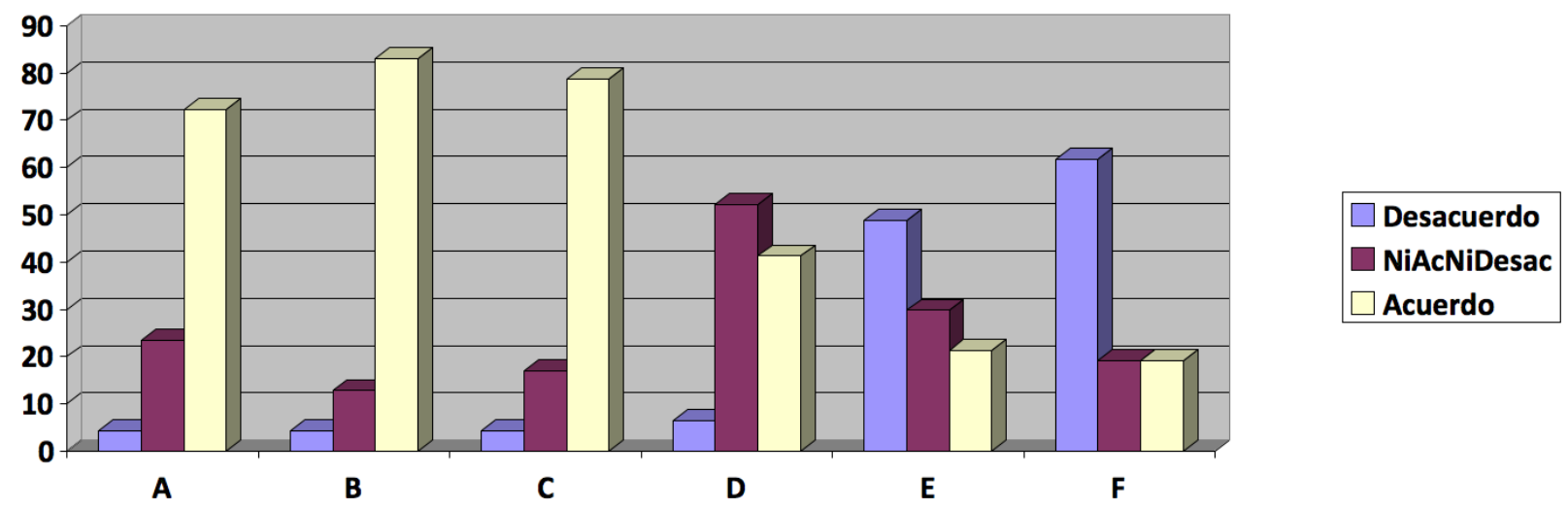

Figura 6. Porcentaje de frecuencias de respuestas para cada afirmación relacionada con los beneficios o motivaciones positivas para utilizar Internet: a) Internet me resulta útil, b) Internet me ayuda a estar actualizado, c) Internet es fácil de usar, d) Wikipedia me parece tanto o más fiable que otras fuentes distintas a Internet,

e) He conocido a nuevas personas gracias a Internet, f) Me parece más fácil expresar algunas cosas por Internet que cara a cara

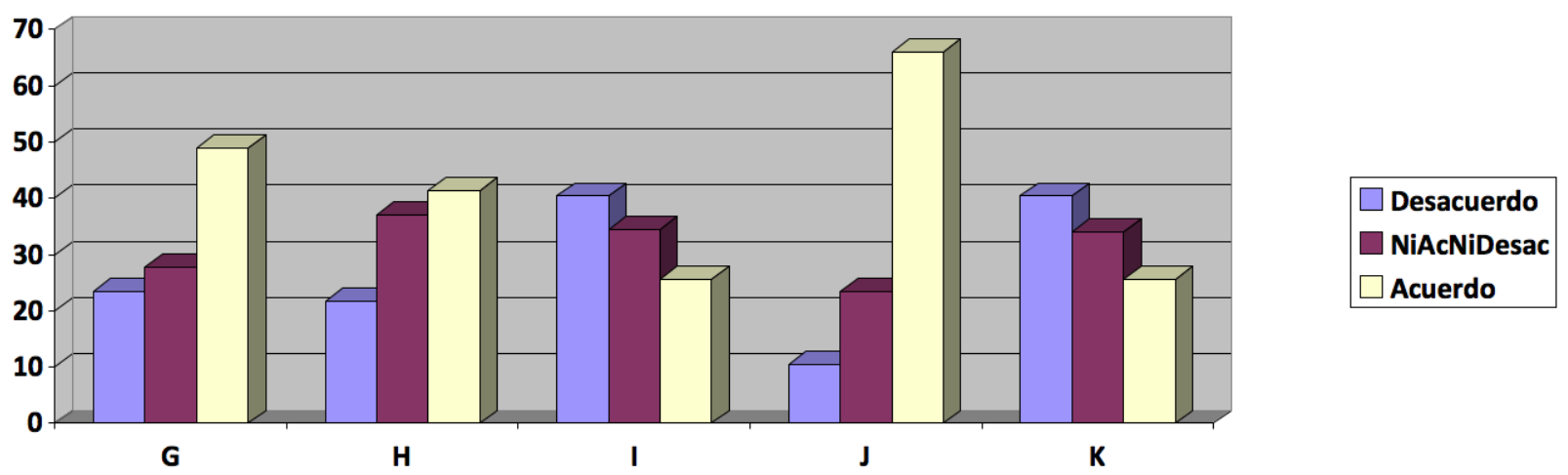

Figura 7. Porcentaje de frecuencias de respuestas para cada afirmación relacionada con los beneficios o motivaciones positivas para utilizar Internet: $g$ ) Me gusta el anonimato de la red, h) En Internet encuentro gente con inquietudes similares a las mías, i) Internet me ayuda a mantener la amistad con otras personas, j) Me preocupa la confidencialidad en Internet, $k$ ) No puedo imaginar la vida sin Internet
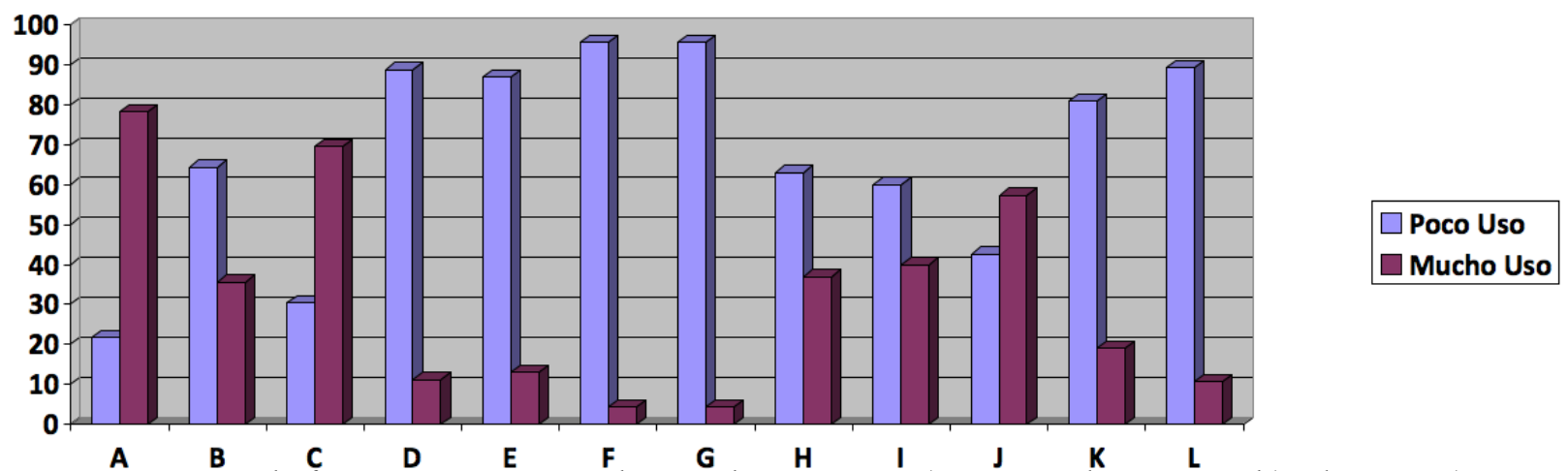

Figura 8: Porcentaje de frecuencias para cada uso de Internet: a) Correo electrónico, b) Chatear, c) Buscar información, d) Ver páginas eróticas, e) Descarga de música, películas, etc., f) Consulta de horóscopo, g) Tarot, h) Gestiones con la Administración pública, i) Fines académicos: clases, formación, j) Leer prensa, k) Banca en línea, l) Reservas para actividades de ocio 


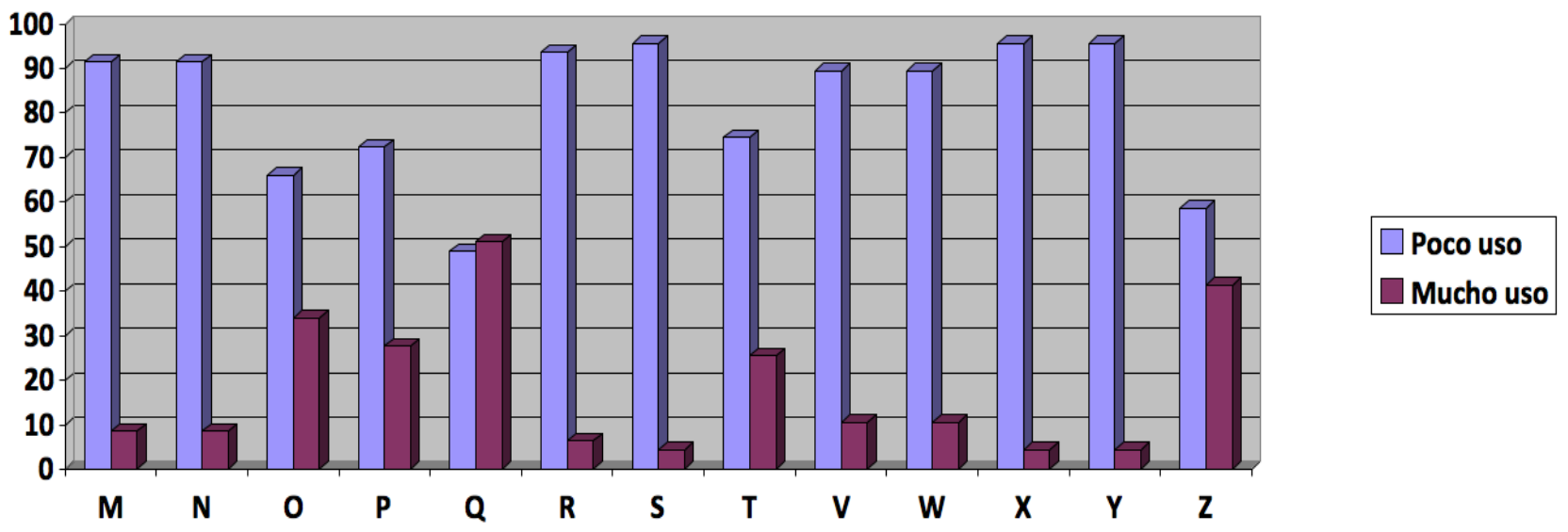

Figura 9: Porcentaje de frecuencias para cada uso de Internet: $m$ ) Comprar/vender, $n$ ) Participar en foros, o) Jugar videojuegos, p) Compartir fotos, archivos, q) Navegar sin ningún propósito en concreto, r) Escuchar la radio, s) Participar en grupos u organizaciones, t) Colgar contenidos en la web, v) Hacer llamadas, w) Videoconferencia, x) Ver la Tv, y) Subastas (eBay, etc.), z) Buscar información sobre temas médicos

consideramos que los programas universitarios de mayores pueden realizar una importante contribución al respecto. En este sentido, podrían facilitar cursos que comiencen con actividades prácticas, útiles y que coincidan con las más empleadas por los mayores, como la búsqueda de información, utilización de correo electrónico,... y continuar progresivamente hacia funciones más avanzadas. Consideramos necesaria la realización de más estudios en diversos países europeos que permita conocer la situación en cuanto a la relación de los mayores activos con la tecnología y la comparación entre los mismos.

\section{Referencias}

Aguaded, J. I. (2010). The European Union passes a recommendation on media literacy in the European digital environment. Comunicar, 34, 7-8.

DiMaggio, P., Hargittai, E., Neuman, W. R. y Robinson, J. P. (2001). Social implications of the internet. Annual Review of Sociology, 27; 307-336.

Hernández-Encuentra, E.; Pousada, M. y Gomez-Zuñiga, B (2009). ICT and older people: beyond usability. Educational Gerontology, 35, 226-245.

Martinez-Pecino, R., Cabecinhas, R. y Loscertales-Abril, F. (2011). University senior students on the web. Comunicar, 37, 89-95.

Selwyn, N., Gorard, S., Furlong, J. y Madden, L. (2003). Older adults' use of information and communications technology in everyday life. Ageing and Society, 23, 561-582. 